The University of Maine

\title{
DigitalCommons@UMaine
}

Maine-Syracuse Longitudinal Papers

Maine-Syracuse Longitudinal Study

2004

\section{New Norms for a New Generation: Cognitive Performance in the Framingham Offspring Cohort}

Rhoda Au

Sudha Seshadri

Philip A. Wolf

Merrill F. Elias

University of Maine - Main, mfelias@maine.edu

Penelope K. Elias

See next page for additional authors

Follow this and additional works at: https://digitalcommons.library.umaine.edu/ longitudinal_papers

Part of the Cognitive Psychology Commons

\section{Repository Citation}

Au, R., Seshadri, S., Wolf, P. A., Elias, M. F., Elias, P. K., Sullivan, L., Beiser, A., \& D'Agostino, R. B. (2004). New norms for a new generation: Cognitive performance in the Framingham offspring cohort. Experimental Aging Research, 30, 333-358.

This Article is brought to you for free and open access by DigitalCommons@UMaine. It has been accepted for inclusion in Maine-Syracuse Longitudinal Papers by an authorized administrator of DigitalCommons@UMaine. For more information, please contact um.library.technical.services@maine.edu. 
Authors

Rhoda Au, Sudha Seshadri, Philip A. Wolf, Merrill F. Elias, Penelope K. Elias, Lisa Sullivan, Alexa Beiser, and Ralph B. D'Agostino 


\title{
New Norms for a New Generation: \\ Cognitive Performance in the Framingham Offspring Cohort
}

\author{
Rhoda Au, Sudha Seshadri, and Philip A. Wolf \\ Department of Neurology, Boston University School \\ of Medicine, Boston, Massachusetts, USA
}

\author{
Merrill F. Elias and Penelope K. Elias \\ Department of Mathematics and Statistics, \\ Boston University, Boston, Massachusetts, USA; \\ and Department of Psychology, University of Maine, \\ Orono, Maine, USA
}

\section{Lisa Sullivan and Alexa Beiser \\ Department of Biostatistics, Boston University \\ School of Public Health, Boston, Massachusetts, USA}

\author{
Ralph B. D'Agostino \\ Department of Mathematics and Statistics, Boston \\ University, Boston, Massachusetts, USA
}

Received 15 July 2003; accepted 11 January 2004.

This work was supported by the Framingham Heart Study's National Heart, Lung, and Blood Institute contract (N01-HC-25195) and by grant (5R01-AG16495-03) from the National Institute on Aging and by grants (5R01-NS17950-19 and 5R01-AG 08122) from the National Institute of Neurological Disorders and Stroke. The authors thank their dedicated team that assisted in testing, including Megan Young, Mary Darling, Nicholas Chechile, Tasha Cooper, Karen Van, Manen, PhD, Sherral Devine, PhD, Michael Tocco, Kimberly Sullivan, PhD, Jessica Chung, and Maureen Valentino. Additional thanks are given to Jessica Chung for her help in preparing the tables.

Address correspondence to Rhoda Au, Department of Neurology, Boston University School of Medicine, 715 Albany Street, B-608, Boston, MA 02118-2526, USA. E-mail: rhodaau@bu.edu 
A previous publication presented normative data on neuropsychological tests stratified by age, gender, and education based on the Original Cohort of the Framingham Heart Study. Many contemporary investigations include subject samples with higher levels of education, a factor known to affect cognitive performance. Secular change in education prompted the reexamination of norms in the children of the Original Cohort. The study population consisted of 853 men and 988 women from the Offspring Study, free of clinical neurological disease, who underwent a neuropsychological examination, which included tests given to their parents in 1974 to 1976 as well as additional newer tests to provide a more comprehensive battery. The Offspring population overall was more evenly distributed by gender and better educated. Their performance on cognitive tests was superior to that of the Original Cohort. Multivariable analyses revealed that more years of education explained only a part of the cohort differences. These findings suggest that continued surveillance of each generation is necessary to document the impact that unique social and economic variables have on cognitive function. Here, the authors provide updated normative data.

Aging is a continuous process involving well-documented changes in cognitive functioning. In addition to age, factors shown to influence cognitive performance include education and gender (Wiederholt et al., 1993; Farmer, Kittner, Rae, Bartko, \& Regier, 1995; Manly et al., 1999; Cagney \& Lauderdale, 2002). Elias, Elias, D’Agostino, Silbershatz, and Wolf (1997) published norms for commonly used neuropsychological tests stratified by age, gender, and education for the populationbased Framingham Original cohort. These data came from assessments done between 1974 and 1976 and the normative tables remain useful for evaluating subjects from this generation. However, their utility for a more contemporary sample involving persons with higher education is limited.

Higher education has been shown repeatedly to be positively correlated with cognitive test performance (Birren \& Morrison, 1961; Cagney \& Lauderdale, 2002; Christensen et al., 1997; Reitan and Wolfson, 1995.) Today far greater percentages of the population possess a high school diploma, and many more go on to complete college than in previous generations. Hence, norms derived from older subjects, who have had less formal education (e.g., Elias et al., 1997), may underestimate normal ranges of performance for those who are younger and more educated. 
The need for new normative data is further underscored by current research on early detection of Alzheimer's disease. These studies deal with the challenge of distinguishing cognitive decline associated with normal aging from deficit performance indicative of prodromal dementia. Motivating these studies is the premise that treatment options for attenuating the rate of progression are seen to be most promising in the initial states of the disease (Doraiswamy, Steffens, Pitchumoni, \& Tabrizi, 1999; Gauthier, 1999.) Most recent efforts to define mild cognitive impairment (MCI), presumed to be the earliest stages of Alzheimer's disease rely, in part, on cognitive measures that fall 1.5 standard deviations below age and education adjusted means on one or more tests (Petersen et al., 1999; 2001) Thus, identification of early-onset dementia is dependent on normative data that reflects contemporary levels of education, lifestyles, recent personal habits, and health education and utilization trends.

The Framingham Study is unique for its extensive surveillance of two community-based cohorts. The Framingham Offspring population is comprised of the progeny of the Original cohort and their spouses. The educational level of this progeny group differs significantly from that of their parents, portending distinctive patterns of cognitive performance.

In addition to the primary goal of providing normative data for a current generation of individuals, the purpose of this study was to test the hypothesis that increased levels of educational attainment in the Offspring Cohort would be related to better performance on neuropsychological tests as compared to the Original Cohort. Expected results motivate the need for updated descriptive-normative data on tests of cognition for a new generation of normal aging individuals who more accurately reflect the impact of secular changes in education on cognition. The potential value of these data will be to facilitate investigations of normal aging and disease populations that rely on subjects also drawn from this era. Further, these data will provide normative information that is necessary to the definition of various indices of cognitive deficits, including MCI.

\section{METHODS}

\section{Subjects}

The Offspring Cohort, recruited in 1971, has undergone seven periodic physical and medical examinations over a 30 -year period to identify risk factors for cardiovascular and cerebrovascular diseases (Garrison, Kannel, Stokes, \& Castelli, 1987). The key criterion for enrollment in the Offspring Study was that at least one of their biological parents or 
their spouse's parents was a member of the Original Cohort. The initial Offspring Cohort consisted of 5124 men and women; $88 \%$ of survivors (3539 of 4031) participated in Examination 7, the current examination cycle.

As part of a large ancillary study on brain magnetic resonance imaging (MRI) and cognitive functioning, Offspring were recruited to take a neuropsychological test (NP) battery. From March 1999 through December 2000, 2187 subjects agreed to undergo neuropsychological testing, of which 1889 also had an MRI. We limited our sample to these 1889 subjects because MR imaging provided data necessary to identify subjects with preexisting neurological disorders. We eliminated 47 subjects who had a clinical stroke $(n=28)$, diagnoses of probable dementia $(n=2)$, multiple sclerosis $(n=6)$, or other neurological illnesses $(n=11)$. We determined if a clinical stroke or probable dementia was present through a consensus review process described in detail in Sacco, Wolf, Kannel, and McNamara (1982) and Bachman et al., (1992), respectively. We excluded one subject who lacked educational attainment information. After all exclusions, our total study size was $1841 ; 853$ men and 988 women.

Comparison of the study sample (MRI + NP) to subjects who took the neuropsychological examination and did not have an MRI (NP-only) found that the NP-only group was older (62.1 years versus 60.6 years for MRI + NP, $p<.002$.$) , had higher prevalence of disease (\%$ with hypertension, $p<.01 ; \%$ myocardial infaraction, $p<.02 ; \%$ cardiovascular disease [CVD], $p=.0001 ; \%$ coronary heart disease [CHD], $p=.02$ ), and were heavier (body mass index [BMI], $p=.002$ ) There were no differences in educational attainment $(p=.33)$ or gender distribution $(p=.75$. $)$

\section{Test Battery}

The neuropsychological test battery consists of most of the tests given to the Original Cohort in 1976 to 1978. Although newer versions of these tests exist today, we continued to use the original versions because this strategy would allow parent/offspring comparisons in the future. Five tests were appended to the Original Cohort battery. They provide a more comprehensive neuropsychological profile and are known to be sensitive to subtle changes in cognitive status. The original and additional tests include the following.

\section{Neuropsychological Protocol: Tests from the Original Cohort}

Logical memory-Immediate and delayed recall. The Logical Memory subtest from the Wechsler Memory Scale (WMS) is one of the most widely used measures of verbal memory, The administration of the delayed recall test provides a savings measure for long-term memory. 
Visual reproduction-Immediate recall. Memory for visual information relies on different processing/storage mechanisms than for verbal materials. Gfeller, Meldru, and Jacobi (1995) reported subjects with constructive impairment performed poorly on the Wechsler Memory Scale-Revised (WMS-R) Visual Reproduction test compared to controls, but showed no difference in performance on Logical Memory. In the Framingham test battery, visual test items come from the WMS visual reproduction subtest; the test protocol used is the same as the WMS-R.

Paired associate learning. Inability to learn new information is a hallmark sign of Alzheimer's disease (AD) and is particularly effective in detecting early symptoms of disease in those of higher education and intelligence. Changes in ability to acquire new information also occur with normal aging. The Paired Associate Learning subtest from the WMS is a well-recognized measure of new learning.

Digit span-Forward and backward. Widely used tests for attention, the difference score between forward and backward spans allow differentiation between impairment in simple attention versus working memory. Caffarra et al., (1991) found that the Wechsler Adult Intelligence Scale (WAIS) score for digit span, the version used in the Framingham Study, was one of only three subtests that significantly differentiated $\mathrm{AD}$ from other types of dementia.

Similarities. Taken from the WAIS, the Similarities test is one of the most frequently used measures of abstract reasoning skills. It also represented one of the other three subtests that Caffara et al., (1991) found to be significantly effective for differential diagnosis of dementias.

\section{Neuropsychological Protocol: Tests in Addition to Original Cohort Battery}

Visual reproductions-Delayed recall. Given that there are modality specific findings for immediate and delayed memory recall, it is important to include the delayed version for visual memory, which we measure for verbal memory. Frank, Wiederholt, Kritz-Silverstein, Salmon, and Barrett-Connor (1996) found that the savings score of the Visual Reproduction test was a sensitive indicator of dementia, and specifically AD.

Verbal memory and visual reproduction-Recognition. Residual memory may exist and a variety of cognitive or motor-related factors may inhibit subjects' ability to verbalize information. Again, modalityspecific recognition skills necessitate measurement of both verbal and visual domains. Stroke-related changes in cognitive functioning may 
often result in deficits in one modality while preserving function in another.

Boston naming test-30 item version. Naming decline associated with normal aging, dementia and stroke are well-documented. The Boston Naming Test (BNT) is the primary measure used to assess naming ability in both normal aging and neurologic populations. Shortened versions of the original 60 -item versions have been tested for equivalency to the 60-item score. Mack, Freed, Williams, and Henderson (1992) found that four different 30 -item versions were comparable to the 60 -item test. The Consortium to Establish a Registry for AD (CERAD) uses a 15-item version that Mack et al., did not find as accurate as the 30 - and 60 -item tests.

Trail making tests $A$ and $B$. Commonly used as a test of visualmotor and attention, Trails A measures simple attention, whereas Trails $\mathrm{B}$ tests cognitive flexibility and a more complex level of attention. The contribution of each subtest as a valid measure of visuomotor and attention skills has been most recently supported by Gaudino, Geisler, and Squires (1995) and Giovagnoli et al., (1996).

Finger tapping test. Frequently used as a measure of motor speed, the Finger Tapping Test allows assessment of laterality effects in motor function. Shimoyama, Ninchoji, and Uemura (1990) found finger tapping in the dominant and nondominant fingers to be sensitive to normal aging effects. Ruff and Parker (1993) report gender effects as well. In the neurologic population, the Finger Tapping Test is an effective measure for detecting motor dysfunction (Arceneaux, Kirkendall, Hill, Dean, \& Anderson, 1997.)

Hooper visual organization test. As a measure of visuoperceptual skills, the Hooper Visual Organization Test (VOT) is the standard neuropsychological test, validated by numerous studies (Boyd, 1981; Gerson, 1974). Lezak (1983) noted that this test is a good tool for detecting right frontal lesions that often result in perceptual fragmentation.

Wide range achievement test - 3, reading. The slope of the decline in cognitive performance is one factor that differentiates normal aging from the early and middle stages of dementia. Determining the degree and rate of decline depends on accurate estimates of premorbid intelligence. Reading words accurately is one validated measure of premorbid intelligence (Filley and Cullum, 1997; Schmand, et al. 1998). It is one of the most widely used methods because reading words is also one of the few skills preserved at the moderate-to-severe stages of AD (Bushell and Martin, 1997; Fleishman et al., 1997; Monti et al., 1997). The Wide Range Achievement 
Test - 3 (WRAT-3) reading subtest is often used as an appropriate "hold" test (Johnstone \& Wilhelm, 1996).

\section{Procedure}

Subjects were administered a neuropsychological test battery as part of a larger study establishing baseline measures of brain structure and cognition in the Offspring Cohort. Standard test administration protocols were used for each test and given by trained examiners. Table 1 provides a list of the ranges of scores for each test. In general, increased scores reflect higher performance. The exception is the timed measures of Trails A and B, where lower scores indicate better performance.

TABLE 1 Range of Scores on Neuropsychological Test Battery

\begin{tabular}{|c|c|c|}
\hline Cognitive domain & Test measure & Range \\
\hline \multirow[t]{3}{*}{ Verbal memory } & $\begin{array}{l}\text { Logical Memory-Immediate } \\
\text { Recall (LM-IR) }\end{array}$ & $0-24$ \\
\hline & $\begin{array}{l}\text { Logical Memory_-Delayed } \\
\text { Recall (LM-DR) }\end{array}$ & $0-24$ \\
\hline & $\begin{array}{l}\text { Logical Memory-\% Retained } \\
\text { Information (LM- } \% \text { ) }\end{array}$ & $0-100$ \\
\hline \multirow[t]{3}{*}{ Visual memory } & $\begin{array}{l}\text { Visual Reproductions-Immediate } \\
\text { Recall (VR-IR) }\end{array}$ & $0-14$ \\
\hline & $\begin{array}{l}\text { Visual Reproductions-Delayed } \\
\text { Recall (VR-DR) }\end{array}$ & $0-14$ \\
\hline & $\begin{array}{l}\text { Visual Reproductions-\% } \% \text { Retained } \\
\text { Information (VR-\%) }\end{array}$ & $0-100$ \\
\hline Learning & $\begin{array}{l}\text { Paired Associates_-Total } \\
\text { Score (PA-TS) }\end{array}$ & $0-21$ \\
\hline \multirow{2}{*}{$\begin{array}{l}\text { Attention and } \\
\text { concentration }\end{array}$} & Trails A-Time & $0-300$ \\
\hline & Trails B-Time & $0-300$ \\
\hline Abstract reasoning & $\begin{array}{l}\text { Similarities-Total Raw } \\
\text { Score (Sim) }\end{array}$ & $0-26$ \\
\hline Language & $\begin{array}{l}\text { Boston Naming Test-Total Correct } \\
\text { ithout Cues (BNT) }\end{array}$ & $0-36$ \\
\hline $\begin{array}{l}\text { Visuoperceptual } \\
\text { organization }\end{array}$ & Hooper-Total Score & $0-30$ \\
\hline Psychomotor speed & Finger Tapping-Dominant Hand Mean (FT-D) & $0-70+$ \\
\hline $\begin{array}{l}\text { Premorbid } \\
\text { intelligence }\end{array}$ & WRAT_Raw Score & $0-57$ \\
\hline
\end{tabular}




\section{Statistical Analysis}

In the first phase of our analysis, we compared the Offspring Cohort to the Original Cohort with respect to age, gender, and education. Of primary interest was the difference in educational levels between groups because of the well-documented impact of education on neuropsychological test performance. Organizing the Offspring Cohort into the same educational groups the Elias et al., study (1997) used in the analysis of the Original Cohort, we conducted a chi-square test to evaluate cohort difference in educational attainment.

In the next phase of the analysis, we replicated the analyses done by Elias et al., (1997), examining the effects of age, educational level, and gender on the battery of cognitive function tests considered in the Offspring Study. We assessed the proportion of variance (e.g, $R^{2}$ ) explained by age, educational level, and gender using multiple regression analysis.

Finally, normative tables follow that describe the distributional properties of each measure for specific age and educational groups in men and women; we present the mean, standard deviation, the first $(25 \%)$ and third $(75 \%)$ quartile scores, and the 5th percentiles scores. The quartile scores reflect performance scores at the 25 th and 75 th percentiles, respectively, within an age, education, and gender group. Similarly, the 5 th percentile scores represent performance for the lowest $5 \%$ for specific age, education, and gender categories.

\section{RESULTS}

\section{Descriptive Comparison of Original Versus Offspring Cohorts}

Age, gender, and education distributions were different in the Offspring Cohort as compared the Original Cohort. At the time of their neuropsychological evaluations, the Offspring were younger than their parents (60.6 years [SD 9.4] versus 67.4 years [SD 7.5], respectively, $p=.0001$.) The gender distribution in the Original Cohort was skewed toward women $(58.6 \%)$ as compared to men $(41.4 \%)$. Within the Offspring Cohort, although women still outnumbered men, the difference was smaller (i.e., $53.2 \%$ versus $46.8 \%$, respectively, $p=.0007$.)

As seen in Table 2, there has been an upward shift in education attained among Offspring as compared to the Original Cohort. In fact, of the $64.1 \%$ of Offspring who went beyond high school, $38.4 \%$ had a college degree or higher. The educational levels of the Offspring were significantly different from those of the Original Cohort $(p<.0001)$. In the Original Cohort, $21.7 \%$ of subjects reported education of less than 9 years and $16.8 \%$ had 9 to 11 years of education. In the Offspring, 
TABLE 2 Distribution of Subjects by Education: Original and Offspring Cohorts

\begin{tabular}{lccccccc}
\hline & \multicolumn{7}{c}{ Education (grade level) } \\
\cline { 2 - 8 } $\begin{array}{l}\text { Cohort } \\
\text { group }\end{array}$ & $<9$ & $9-11$ & 12 & $\begin{array}{c}\text { Some } \\
\text { college }\end{array}$ & $\begin{array}{c}\text { College } \\
\text { graduate }\end{array}$ & $>$ College & Total \\
\hline Original & 436 & 338 & 593 & 391 & 148 & 100 & 2006 \\
& $21.7 \%$ & $16.8 \%$ & $29.6 \%$ & $19.5 \%$ & $7.4 \%$ & $5.0 \%$ & \\
Offspring & 12 & 56 & 592 & 472 & 356 & 351 & 1839 \\
& $0.6 \%$ & $3.0 \%$ & $32.3 \%$ & $25.7 \%$ & $19.4 \%$ & $19.0 \%$ & \\
\hline
\end{tabular}

few subjects fell into these low-education categories. Table 3 depicts the age and education distributions by gender for the Offspring Cohort.

\section{Multiple Regression Analysis of Offspring Data}

Unlike findings reported in Elias et al., (1997), we found no significant interactions between age, gender, and education on cognitive function. When we examined the proportion of variance accounted for by age and education, we found similar patterns for all test measures that were

TABLE 3 Age and Education Distributions by Gender: Offspring Cohort

\begin{tabular}{lcccc}
\hline & \multicolumn{4}{c}{ Age at testing } \\
\cline { 2 - 5 } Education & $30-54$ & $55-64$ & $65-74$ & $75+$ \\
\hline Men & & & \\
High School graduate or less & 47 & 85 & 110 & 35 \\
& $(21.0 \%)$ & $(27.7 \%)$ & $(42.8 \%)$ & $(47.9 \%)$ \\
Some or college graduate & 100 & 121 & 80 & 24 \\
& $(44.6 \%)$ & $(39.4 \%)$ & $(31.1 \%)$ & $(32.9 \%)$ \\
Postgraduate or more & 77 & 101 & 67 & 14 \\
& $(34.4 \%)$ & $(32.9 \%)$ & $(26.1 \%)$ & $(19.2 \%)$ \\
Women & 74 & 129 & 135 & 45 \\
High School graduate or less & $(26.7 \%)$ & $(38.9 \%)$ & $(47.7 \%)$ & $(52.3 \%)$ \\
Some or college graduate & 108 & 100 & 69 & 20 \\
Postgraduate or more & $(39.0 \%)$ & $(30.1 \%)$ & $(24.4 \%)$ & $(23.3 \%)$ \\
& 95 & 103 & 79 & 21 \\
& $(34.3 \%)$ & $(31.0 \%)$ & $(27.9 \%)$ & $(24.4 \%)$ \\
\hline
\end{tabular}


administered to both the Offspring and Original Cohorts (see Table 4). The one exception was for Logical Memory-Retained Information. In analysis of the Original Cohort, education was significant, whereas in this analysis of the Offspring Cohort, we found that education did not explain a statistically significant amount of variance $\left(R^{2}=.003, p=.0671\right)$.

Table 5 shows the proportion of variance accounted for by age, education, and gender for each test measured in the Offspring Cohort. With the exception of the Logical Memory-Retained Information and Visual Reproduction measures, age and education each had a small but significant impact on test performance. Only age was significant for Logical Memories-Retained Information. Age and education did account for a significant amount of variance for Visual Reproductions, but gender did not.

\section{Comparison of Original and Offspring Test Performance}

Using multiple regression analysis, we compared performance on the subset of neuropsychological tests that were given to both cohorts. Our initial model included cohort (i.e., Original and Offspring), age, gender, and education. As shown in Table 6a, the initial model and its components were significant for all tests $(p<.0001$.) Offspring test performance was significantly better than the Original Cohort for all measures after adjustment for age, gender, and educational level. Education was the most significant covariate and the model explained $>40 \%$ of the difference in test performance between the Offspring and Original Cohorts.

TABLE 4 Offspring and Original Cohort Comparisons of Zero-Order Associations for Test Measures

\begin{tabular}{|c|c|c|c|c|}
\hline \multirow[b]{2}{*}{ Test measure } & \multicolumn{2}{|c|}{$R^{2}$-age } & \multicolumn{2}{|c|}{$R^{2}$ - education } \\
\hline & Offspring & Original & Offspring & Original \\
\hline $\begin{array}{l}\text { Logical Memory- } \\
\text { Immediate Recall }\end{array}$ & $.04^{* * *}$ & $.06^{* * *}$ & $.08^{* * *}$ & $.09^{* * *}$ \\
\hline $\begin{array}{l}\text { Logical Memory- } \\
\text { Delayed Recall }\end{array}$ & $.05^{* * *}$ & $.08^{* * *}$ & $.07^{* * *}$ & $.07^{* * *}$ \\
\hline $\begin{array}{l}\text { Logical Memory- } \\
\text { Retained Information }\end{array}$ & $.02^{* * *}$ & $.04^{* * *}$ & .003 & $.01^{* *}$ \\
\hline $\begin{array}{l}\text { Visual Reproductions- } \\
\text { Immediate Recall }\end{array}$ & $.13^{* * *}$ & $.02^{* * *}$ & $.08^{* * *}$ & $.05^{* * *}$ \\
\hline Paired Associates & $.07^{* * *}$ & $.06^{* * *}$ & $.04^{* * *}$ & $.02^{* * *}$ \\
\hline Similarities & $.09^{* * *}$ & $.07^{* * *}$ & $.16^{* * *}$ & $.18^{* * *}$ \\
\hline
\end{tabular}

${ }^{*} p<.01 ;{ }^{* *} p<.001 ;{ }^{* * *} p<.0001$. 
TABLE 5 Summary of Zero-Order Associations $\left(R^{2}\right)$ Between Test Measure and Age, Education, and Gender

\begin{tabular}{lccc}
\hline Test measure & $R^{2}$-age & $R^{2}$-education & $R^{2}$-gender \\
\hline Logical Memory_Immediate Recall & $.04^{* * *}$ & $.08^{* * *}$ & $.01^{* * *}$ \\
Logical Memory_Delayed Recall & $.05^{* * *}$ & $.07^{* * *}$ & $.01^{* * *}$ \\
Logical Memory-Retained Information & $.02^{* * *}$ & .003 & .000 \\
Visual Reproductions-Immediate Recall & $.13^{* * *}$ & $.08^{* * *}$ & .002 \\
Visual Reproductions-Delayed Recall & $.14^{* * *}$ & $.08^{* * *}$ & .001 \\
Visual Reproductions-Retained & $.02^{* * *}$ & .003 & .0004 \\
$\quad$ Information & $.07^{* * *}$ & $.04^{* * *}$ & $.06^{* * *}$ \\
Paired Associates & $.09^{* * *}$ & $.16^{* * *}$ & .0003 \\
Similarities & $.08^{* * *}$ & $.06^{* * *}$ & $.005^{*}$ \\
Boston Naming & $.12^{* * *}$ & $.02^{* * *}$ & $.005^{*}$ \\
Trails A & $.17^{* * *}$ & $.06^{* * *}$ & .001 \\
Trails B & $.14^{* * *}$ & $.02^{* * *}$ & $.006^{*}$ \\
Hooper & $.11^{* * *}$ & $.02^{* * *}$ & $.12^{* * *}$ \\
Finger Tapping-Dominant Hand & $.03^{* * *}$ & $.24^{* * *}$ & $.006^{* *}$ \\
WRAT-Reading & & & \\
\hline
\end{tabular}

$$
{ }^{*} p<.01 ;{ }^{* *} p<.001 ;{ }^{* * *} p<.0001 .
$$

Although these statistical adjustments affected mean performance, comparison with unadjusted means indicates these variables do not fully explain the differences between the two cohorts (see Table 6b).

To address residual error we added to this model (1) occupation, (2) two-way interactions of gender and education and gender and occupation, and (3) the three-way interaction of gender, education, and occupation. Neither occupation nor the interactions significantly increased the $R^{2}$ (Table 6c).

TABLE 6a Multivariable Analyses: Initial Model

\begin{tabular}{lccrrr}
\hline \multicolumn{5}{c}{ Neuropsychological tests } \\
\cline { 2 - 6 } & LM-IR & LM-DR & VR-IR & PA-TS & Sim \\
\hline Model & $<.0001$ & $<.0001$ & $<.0001$ & $<.0001$ & $<.0001$ \\
Cohort & $<.0001$ & $<.0001$ & $<.0001$ & .0307 & $<.0001$ \\
Age & $<.0001$ & $<.0001$ & $<.0001$ & $<.0001$ & $<.0001$ \\
Gender & .0003 & .0286 & .2862 & $<.0001$ & .7641 \\
Education & $<.0001$ & $<.0001$ & $<.0001$ & $<.0001$ & $<.0001$ \\
$R^{2}$ & .409 & .447 & .352 & .197 & .480 \\
\hline
\end{tabular}


TABLE 6b Multivariable Analyses

\begin{tabular}{lccccc}
\hline & \multicolumn{5}{c}{ Neuropsychological tests } \\
\cline { 2 - 6 } & LM-IR & LM-DR & VR-IR & PA-TS & Sim \\
\hline Unadjusted means (SD) & & & & & \\
Original & 6.98 & 5.68 & 5.98 & 12.29 & 11.72 \\
& $(3.44)$ & $(3.51)$ & $(3.14)$ & $(3.46)$ & $(5.72)$ \\
Offspring & 11.36 & 9.51 & 9.02 & 13.82 & 16.75 \\
& $(3.43)$ & $(1.22)$ & $(3.20)$ & $(3.34)$ & $(3.60)$ \\
Adjusted means (SE) & & & & & \\
Original & 7.68 & 6.10 & 6.75 & 12.75 & 12.99 \\
& $(0.08)$ & $(0.06)$ & $(0.07)$ & $(0.08)$ & $(0.10)$ \\
Offspring & 10.40 & 8.70 & 8.12 & 13.01 & 14.75 \\
& $(0.08)$ & $(0.07)$ & $(0.08)$ & $(0.08)$ & $(0.11)$ \\
\hline
\end{tabular}

TABLE 6c Multivariable Analyses: $R^{2}$ for Occupation and Interactions

\begin{tabular}{lccccc}
\hline & \multicolumn{5}{c}{ Neuropsychological tests } \\
\cline { 2 - 6 } & LM-IR & LM-DR & VR-IR & PA-TS & Sim \\
\hline Occupation & .416 & .450 & .359 & .201 & .488 \\
Gender $\times$ Education & .419 & .453 & .364 & .203 & .492 \\
Gender $\times$ Education $\times$ Occupation & .431 & .460 & .375 & .212 & .502 \\
\hline
\end{tabular}

\section{Age, Education, and Gender Norms}

We report below new performance data based on our Offspring Cohort. The tables include measures that were reported in our earlier study and additional newer measures. Table 7 contains the number of subjects, means, standard deviations, upper and lower quartiles, and 5 th percentile scores for each measure stratified by specific age group, educational level, and gender.

\section{DISCUSSION}

The demographics profile for the Framingham Offspring Cohort was different from that of the Original Cohort. In the Offspring, the age range included a younger age cohort. The Offspring population were more evenly distributed by gender, and were better educated. 
TABLE 7 Means and S.D. by Education $\times$ Gender by Age Group

\begin{tabular}{|c|c|c|c|c|c|c|c|c|c|}
\hline & \multicolumn{3}{|c|}{ High school } & \multicolumn{3}{|c|}{ College } & \multicolumn{3}{|c|}{$>$ College } \\
\hline & All & Men & Women & All & Men & Women & All & Men & Women \\
\hline \multirow{2}{*}{\multicolumn{10}{|c|}{$\begin{array}{l}\text { 30- to } 54 \text {-year-old age group } \\
\text { LM-IR }\end{array}$}} \\
\hline & & & & & & & & & \\
\hline$N$ & 116 & 44 & 72 & 201 & 96 & 105 & 166 & 73 & 93 \\
\hline Mean & 10.74 & 9.93 & 11.24 & 12.35 & 11.95 & 12.71 & 13.22 & 12.86 & 13.49 \\
\hline$S D$ & 3.10 & 3.08 & 3.04 & 2.91 & 2.80 & 2.98 & 3.18 & 3.19 & 3.17 \\
\hline Upper Q & 13 & 12 & 13 & 14 & 14 & 15 & 16 & 15 & 16 \\
\hline Lower Q & 9 & 8 & 9 & 10 & 10 & 11 & 11 & 11 & 11 \\
\hline $5 \%$ & 5 & 5 & 6 & 8 & 8 & 7 & 8 & 7 & 8 \\
\hline \multicolumn{10}{|l|}{ LM-DR } \\
\hline$N$ & 115 & 44 & 71 & 201 & 96 & 105 & 166 & 73 & 93 \\
\hline Mean & 9.74 & 9.16 & 10.10 & 11.61 & 11.13 & 12.06 & 12.51 & 11.86 & 13.01 \\
\hline$S D$ & 3.39 & 3.55 & 3.26 & 3.20 & 3.41 & 2.95 & 3.38 & 3.17 & 3.46 \\
\hline Upper Q & 12 & 11 & 12 & 14 & 14 & 14 & 15 & 14 & 15 \\
\hline Lower Q & 8 & 7 & 8 & 9 & 9 & 10 & 10 & 10 & 11 \\
\hline $5 \%$ & 3 & 3 & 5 & 7 & 6 & 8 & 7 & 6 & 7 \\
\hline \multicolumn{10}{|l|}{ LM-\% } \\
\hline$N$ & 115 & 44 & 71 & 201 & 96 & 105 & 166 & 73 & 93 \\
\hline Mean & 91.21 & 91.85 & 90.82 & 94.27 & 92.45 & 95.95 & 95.05 & 92.69 & 96.91 \\
\hline$S D$ & 23.61 & 23.76 & 23.68 & 17.44 & 18.49 & 16.33 & 15.79 & 13.11 & 17.45 \\
\hline Upper Q & 100 & 100 & 100 & 100 & 107.42 & 100 & 11 & 100 & 107.14 \\
\hline Lower Q & 81.82 & 83.33 & 80 & 83.33 & 80.63 & 86.67 & 84.62 & 83.33 & 85.71 \\
\hline $5 \%$ & 50 & 44.44 & 58.33 & 66.67 & 60 & 76.92 & 71.43 & 71.43 & 70 \\
\hline \multicolumn{10}{|l|}{ VR-IR } \\
\hline$N$ & 116 & 44 & 72 & 201 & 96 & 105 & 166 & 73 & 93 \\
\hline Mean & 9.46 & 10.16 & 9.03 & 10.71 & 10.69 & 10.72 & 10.91 & 11.19 & 10.69 \\
\hline$S D$ & 2.79 & 2.95 & 2.61 & 2.53 & 2.82 & 2.23 & 2.51 & 2.46 & 2.55 \\
\hline Upper Q & 11 & 13 & 11 & 13 & 13 & 13 & 13 & 13 & 13 \\
\hline Lower Q & 8 & 8 & 8 & 9 & 9 & 9 & 9 & 9 & 9 \\
\hline $5 \%$ & 4 & 5 & 4 & 6 & 6 & 7 & 6 & 7 & 6 \\
\hline \multicolumn{10}{|l|}{ VR-DR } \\
\hline$N$ & 115 & 44 & 71 & 201 & 96 & 105 & 166 & 73 & 93 \\
\hline Mean & 8.74 & 9.34 & 8.37 & 9.87 & 9.74 & 9.99 & 10.29 & 10.23 & 10.33 \\
\hline$S D$ & 2.91 & 2.91 & 2.86 & 3.06 & 3.47 & 2.64 & 2.73 & 2.96 & 2.56 \\
\hline Upper Q & 11 & 12 & 10 & 13 & 13 & 12 & 12 & 13 & 12 \\
\hline Lower Q & 6 & 7 & 6 & 8 & 7 & 9 & 8 & 7 & 9 \\
\hline $5 \%$ & 4 & 5 & 4 & 5 & 4 & 5 & 5 & 5 & 6 \\
\hline \multicolumn{10}{|l|}{ VR-\% } \\
\hline$N$ & 115 & 44 & 71 & 201 & 96 & 105 & 166 & 73 & 93 \\
\hline Mean & 93.16 & 93.17 & 93.15 & 91.58 & 90.11 & 92.92 & 94.36 & 90.49 & 97.39 \\
\hline$S D$ & 17.87 & 17.96 & 17.94 & 17.44 & 18.94 & 15.19 & 14.33 & 13.41 & 14.36 \\
\hline Upper Q & 100 & 100 & 100 & 100 & 100 & 100 & 100 & 100 & 100 \\
\hline Lower Q & 81.82 & 81.82 & 81.82 & 83.33 & 79.29 & 84.62 & 87.5 & 85.71 & 90.91 \\
\hline $5 \%$ & 60 & 60 & 62.5 & 60 & 50 & 60 & 69.23 & 62.5 & 72.73 \\
\hline
\end{tabular}


TABLE 7 Continued

\begin{tabular}{|c|c|c|c|c|c|c|c|c|c|}
\hline & \multicolumn{3}{|c|}{ High school } & \multicolumn{3}{|c|}{ College } & \multicolumn{3}{|c|}{$>$ College } \\
\hline & All & Men & Women & All & Men & Women & All & Men & Women \\
\hline \multicolumn{10}{|l|}{ PA-TS } \\
\hline$N$ & 115 & 44 & 71 & 201 & 96 & 105 & 166 & 73 & 93 \\
\hline Mean & 13.91 & 13.41 & 14.23 & 15.12 & 14.71 & 15.50 & 15.61 & 15.11 & 16 \\
\hline$S D$ & 3.36 & 3.16 & 3.46 & 3.12 & 3.22 & 2.98 & 2.63 & 2.62 & 2.58 \\
\hline Upper Q & 16 & 16 & 17 & 18 & 17 & 18 & 18 & 17 & 18 \\
\hline Lower Q & 12 & 11.5 & 12 & 13 & 12 & 14 & 14 & 14 & 14 \\
\hline $5 \%$ & 8 & 9 & 8 & 9 & 9 & 10 & 11 & 10 & 11 \\
\hline \multicolumn{10}{|l|}{ Trails A } \\
\hline$N$ & 115 & 44 & 71 & 201 & 96 & 105 & 166 & 73 & 93 \\
\hline Mean & 28.43 & 30.43 & 27.20 & 27.62 & 27.65 & 27.59 & 26.20 & 26.55 & 25.94 \\
\hline$S D$ & 8.56 & 10.13 & 7.22 & 8.46 & 8.53 & 8.44 & 6.98 & 6.97 & 7.02 \\
\hline Upper Q & 32 & 34 & 31 & 32 & 32 & 32 & 30 & 30 & 29 \\
\hline Lower Q & 23 & 23.5 & 22 & 21 & 21 & 22 & 21 & 22 & 21 \\
\hline $5 \%$ & 18 & 20 & 18 & 18 & 18 & 17 & 17 & 17 & 17 \\
\hline \multicolumn{10}{|l|}{ Trails B } \\
\hline$N$ & 115 & 44 & 71 & 200 & 95 & 105 & 165 & 73 & 92 \\
\hline Mean & 70.31 & 76.11 & 66.72 & 62.61 & 64.01 & 61.33 & 56.37 & 55.67 & 56.92 \\
\hline$S D$ & 25.37 & 25.94 & 24.5 & 25.40 & 24.57 & 26.18 & 16.03 & 15.36 & 16.61 \\
\hline Upper Q & 82 & 91.5 & 74 & 72 & 72 & 72 & 66 & 67 & 66 \\
\hline Lower Q & 53 & 59 & 51 & 48 & 49 & 48 & 44 & 43 & 45.5 \\
\hline $5 \%$ & 41 & 44 & 37 & 35 & 35 & 35 & 35 & 35 & 33 \\
\hline \multicolumn{10}{|l|}{ Sim } \\
\hline$N$ & 115 & 44 & 71 & 201 & 96 & 105 & 166 & 73 & 93 \\
\hline Mean & 16.28 & 16.39 & 16.21 & 18.30 & 18.44 & 18.14 & 18.95 & 19.19 & 18.75 \\
\hline$S D$ & 2.86 & 3.13 & 2.69 & 2.68 & 2.82 & 2.55 & 2.64 & 2.37 & 2.83 \\
\hline Upper Q & 18 & 19 & 18 & 20 & 20 & 20 & 21 & 21 & 21 \\
\hline Lower Q & 15 & 15 & 15 & 16 & 16 & 17 & 17 & 18 & 17 \\
\hline $5 \%$ & 11 & 12 & 11 & 14 & 14 & 14 & 15 & 15 & 13 \\
\hline \multicolumn{10}{|l|}{ BNT } \\
\hline$N$ & 123 & 47 & 76 & 209 & 100 & 109 & 173 & 78 & 95 \\
\hline Mean & 27.16 & 27.87 & 26.72 & 28.29 & 28.44 & 28.15 & 28.58 & 28.69 & 28.48 \\
\hline$S D$ & 2.64 & 1.80 & 2.97 & 2.41 & 1.90 & 2.80 & 1.66 & 1.72 & 1.62 \\
\hline Upper Q & 29.0 & 29.0 & 28.0 & 30.0 & 30.0 & 30.0 & 30.0 & 30.0 & 30.0 \\
\hline Lower Q & 26.0 & 27.0 & 26.0 & 28.0 & 27.0 & 28.0 & 28.0 & 28.0 & 27.0 \\
\hline $5 \%$ & 24.0 & 26.0 & 23.0 & 25.0 & 24.5 & 25.0 & 25.0 & 25.0 & 25.0 \\
\hline \multicolumn{10}{|l|}{ Hooper } \\
\hline$N$ & 116 & 44 & 72 & 200 & 96 & 104 & 166 & 73 & 93 \\
\hline Mean & 26.02 & 26.49 & 25.73 & 26.42 & 26.19 & 26.63 & 26.28 & 26.33 & 26.24 \\
\hline$S D$ & 2.23 & 2.14 & 2.24 & 1.97 & 2.22 & 1.70 & 2.17 & 2.46 & 1.93 \\
\hline Upper Q & 27.5 & 28 & 27.5 & 28 & 28 & 28 & 28 & 28 & 28 \\
\hline Lower Q & 24.5 & 25 & 24.5 & 25.5 & 24.5 & 25.5 & 25 & 25 & 25 \\
\hline $5 \%$ & 22 & 22 & 21 & 23 & 22 & 23 & 22.5 & 21 & 23 \\
\hline
\end{tabular}


TABLE 7 Continued

\begin{tabular}{|c|c|c|c|c|c|c|c|c|c|}
\hline & \multicolumn{3}{|c|}{ High school } & \multicolumn{3}{|c|}{ College } & \multicolumn{3}{|c|}{$>$ College } \\
\hline & All & Men & Women & All & Men & Women & All & Men & Women \\
\hline \multicolumn{10}{|l|}{ FT-D } \\
\hline$N$ & 107 & 38 & 69 & 190 & 89 & 101 & 150 & 65 & 85 \\
\hline Mean & 47.97 & 52.05 & 45.72 & 49 & 52.81 & 45.64 & 49.8 & 52.14 & 48.01 \\
\hline$S D$ & 7.60 & 8.03 & 6.37 & 7.39 & 6.36 & 6.58 & 8.13 & 8.15 & 7.69 \\
\hline Upper Q & 53 & 56 & 50 & 49 & 58 & 50 & 56 & 58 & 54 \\
\hline Lower Q & 43 & 50 & 41 & 44 & 49 & 42 & 46 & 48 & 45 \\
\hline $5 \%$ & 36 & 35 & 36 & 36 & 42 & 34 & 38 & 38 & 38 \\
\hline \multicolumn{10}{|l|}{ WRAT } \\
\hline$N$ & 115 & 44 & 71 & 201 & 96 & 105 & 166 & 73 & 93 \\
\hline Mean & 46.00 & 45.20 & 46.48 & 50.31 & 49.77 & 50.81 & 51.70 & 52.10 & 51.40 \\
\hline$S D$ & 4.45 & 5.38 & 3.72 & 4.11 & 4.38 & 3.80 & 4.57 & 3.56 & 5.23 \\
\hline Upper Q & 49 & 49 & 49 & 53 & 53 & 54 & 55 & 55 & 55 \\
\hline Lower Q & 43 & 41 & 44 & 48 & 47 & 49 & 50 & 50 & 50 \\
\hline $5 \%$ & 37 & 36 & 41 & 42 & 41 & 43 & 45 & 45 & 45 \\
\hline \multicolumn{10}{|c|}{ 55- to 64-year-old age group } \\
\hline$N$ & 208 & 81 & 127 & 214 & 119 & 95 & 199 & 100 & 99 \\
\hline Mean & 10.21 & 9.35 & 10.76 & 11.62 & 11.37 & 11.94 & 12.57 & 11.96 & 13.19 \\
\hline$S D$ & 3.21 & 3.26 & 3.07 & 3.14 & 3.10 & 3.17 & 3.12 & 3.18 & 2.95 \\
\hline Upper Q & 13 & 12 & 13 & 13 & 13 & 14 & 15 & 14 & 15 \\
\hline Lower Q & 8 & 7 & 9 & 10 & 9 & 10 & 10 & 10 & 11 \\
\hline $5 \%$ & 4 & 4 & 6 & 6 & 6 & 6 & 7 & 7 & 8 \\
\hline \multicolumn{10}{|l|}{ LM-DR } \\
\hline$N$ & 208 & 81 & 127 & 214 & 119 & 95 & 199 & 100 & 99 \\
\hline Mean & 9.45 & 8.47 & 10.07 & 10.63 & 10.37 & 10.96 & 11.57 & 11.07 & 12.07 \\
\hline$S D$ & 3.34 & 3.11 & 3.34 & 3.17 & 3.08 & 3.27 & 3.26 & 3.21 & 3.25 \\
\hline Upper Q & 12 & 11 & 12 & 13 & 12 & 13 & 14 & 13.5 & 15 \\
\hline Lower Q & 7 & 6 & 8 & 9 & 8 & 9 & 9 & 8.5 & 10 \\
\hline $5 \%$ & 4 & 3 & 5 & 5 & 5 & 5 & 6 & 6.5 & 6 \\
\hline \multicolumn{10}{|l|}{ LM- $\%$} \\
\hline$N$ & 208 & 81 & 127 & 214 & 119 & 95 & 199 & 100 & 99 \\
\hline Mean & 93.11 & 92.52 & 93.48 & 92.33 & 91.96 & 92.79 & 92.14 & 92.42 & 91.87 \\
\hline$S D$ & 20.82 & 26.37 & 16.45 & 18.66 & 17.68 & 19.9 & 17.13 & 17.54 & 16.79 \\
\hline Upper Q & 100 & 100 & 100 & 100 & 100 & 100 & 100 & 100 & 100 \\
\hline Lower Q & 81.82 & 80 & 83.33 & 81.82 & 81.82 & 81.82 & 83.33 & 84.62 & 81.82 \\
\hline $5 \%$ & 61.54 & 61.54 & 66.67 & 62.50 & 61.54 & 63.64 & 64.29 & 70.71 & 60 \\
\hline \multicolumn{10}{|l|}{ VR-IR } \\
\hline$N$ & 208 & 81 & 127 & 214 & 119 & 95 & 199 & 100 & 99 \\
\hline Mean & 8.41 & 8.10 & 8.61 & 9.71 & 9.71 & 9.69 & 9.71 & 9.91 & 9.52 \\
\hline$S D$ & 3.18 & 3.43 & 3.00 & 2.76 & 2.73 & 2.81 & 3.21 & 3.33 & 3.10 \\
\hline Upper Q & 11 & 11 & 11 & 12 & 12 & 12 & 12 & 13 & 12 \\
\hline Lower Q & 6 & 6 & 7 & 8 & 8 & 8 & 8 & 8 & 8 \\
\hline $5 \%$ & 3 & 3 & 3 & 5 & 5 & 4 & 4 & 4.5 & 4 \\
\hline
\end{tabular}


TABLE 7 Continued

\begin{tabular}{|c|c|c|c|c|c|c|c|c|c|}
\hline & \multicolumn{3}{|c|}{ High school } & \multicolumn{3}{|c|}{ College } & \multicolumn{3}{|c|}{$>$ College } \\
\hline & All & Men & Women & All & Men & Women & All & Men & Women \\
\hline \multicolumn{10}{|l|}{ VR-DR } \\
\hline$N$ & 208 & 81 & 127 & 214 & 119 & 95 & 199 & 100 & 99 \\
\hline Mean & 7.39 & 7.16 & 7.54 & 8.82 & 8.67 & 9 & 9.17 & 9.37 & 8.97 \\
\hline$S D$ & 3.06 & 3.49 & 2.75 & 3.06 & 2.99 & 3.16 & 3.23 & 3.29 & 3.18 \\
\hline Upper Q & 10 & 9 & 10 & 11 & 11 & 11 & 12 & 12 & 11 \\
\hline Lower Q & 5 & 5 & 6 & 7 & 7 & 7 & 7 & 7 & 7 \\
\hline $5 \%$ & 2 & 1 & 3 & 4 & 4 & 3 & 3 & 3.5 & 3 \\
\hline \multicolumn{10}{|l|}{ VR-\% } \\
\hline$N$ & 207 & 80 & 127 & 214 & 119 & 95 & 199 & 100 & 99 \\
\hline Mean & 90.72 & 91.20 & 90.42 & 90.97 & 89.47 & 92.85 & 95.38 & 95.86 & 94.89 \\
\hline$S D$ & 31.96 & 36.35 & 29.00 & 18.86 & 19.00 & 18.60 & 21.27 & 24.00 & 18.21 \\
\hline Upper Q & 100 & 100 & 100 & 100 & 100 & 100 & 100 & 100 & 100 \\
\hline Lower Q & 76.92 & 77.35 & 76.92 & 81.82 & 80 & 84.62 & 85.71 & 85.71 & 87.5 \\
\hline $5 \%$ & 50 & 46.43 & 50 & 55.56 & 50 & 60 & 61.54 & 60.77 & 62.5 \\
\hline \multicolumn{10}{|l|}{ PA-TS } \\
\hline$N$ & 208 & 81 & 127 & 213 & 119 & 94 & 199 & 100 & 99 \\
\hline Mean & 13.33 & 11.89 & 14.24 & 13.92 & 12.69 & 15.48 & 14.48 & 13.55 & 15.41 \\
\hline$S D$ & 3.31 & 3.12 & 3.11 & 3.37 & 3.09 & 3.06 & 3.25 & 3.21 & 3.03 \\
\hline Upper Q & 16 & 14 & 17 & 16 & 15 & 18 & 17 & 16 & 18 \\
\hline Lower Q & 11 & 9 & 12 & 11 & 10 & 14 & 12 & 11 & 13 \\
\hline $5 \%$ & 8 & 8 & 9 & 9 & 8 & 10 & 9 & 8 & 10 \\
\hline \multicolumn{10}{|l|}{ Trails A } \\
\hline$N$ & 208 & 81 & 127 & 215 & 120 & 95 & 199 & 100 & 99 \\
\hline Mean & 32.83 & 35.68 & 31.02 & 30.93 & 31.64 & 30.04 & 30.75 & 32.20 & 29.29 \\
\hline$S D$ & 15.04 & 20.70 & 9.53 & 10.05 & 10.88 & 8.87 & 11.93 & 13.34 & 10.16 \\
\hline Upper Q & 36 & 39 & 36 & 36 & 37.5 & 35 & 36 & 37 & 34 \\
\hline Lower Q & 24.5 & 26 & 24 & 24 & 24 & 24 & 22 & 23.5 & 22 \\
\hline $5 \%$ & 20 & 20 & 20 & 19 & 19 & 17 & 18 & 17 & 18 \\
\hline \multicolumn{10}{|l|}{ Trails B } \\
\hline$N$ & 205 & 79 & 126 & 214 & 119 & 95 & 196 & 97 & 99 \\
\hline Mean & 85.08 & 92.53 & 80.41 & 71.55 & 75.13 & 67.06 & 71.06 & 70.18 & 71.93 \\
\hline$S D$ & 34.98 & 36.96 & 32.98 & 25.51 & 27.23 & 22.52 & 28.35 & 29.32 & 27.49 \\
\hline Upper Q & 96 & 112 & 91 & 84 & 87 & 79 & 81.5 & 80 & 84 \\
\hline Lower Q & 61 & 62 & 60 & 56 & 58 & 52 & 51.5 & 51 & 53 \\
\hline $5 \%$ & 46 & 49 & 46 & 37 & 40 & 36 & 39 & 38 & 42 \\
\hline \multicolumn{10}{|l|}{ Sim } \\
\hline$N$ & 208 & 81 & 127 & 215 & 120 & 95 & 199 & 100 & 99 \\
\hline Mean & 15.49 & 15.20 & 15.68 & 17.63 & 17.37 & 17.97 & 18.44 & 18.48 & 18.39 \\
\hline$S D$ & 3.55 & 4.20 & 3.07 & 3.16 & 3.25 & 3.02 & 2.87 & 2.52 & 3.19 \\
\hline Upper Q & 18 & 18 & 18 & 20 & 20 & 20 & 20 & 20 & 20 \\
\hline Lower Q & 14 & 13 & 14 & 16 & 15 & 17 & 16 & 17 & 16 \\
\hline $5 \%$ & 9 & 8 & 10 & 12 & 12 & 13 & 13 & 13.5 & 13 \\
\hline
\end{tabular}


TABLE 7 Continued

\begin{tabular}{|c|c|c|c|c|c|c|c|c|c|}
\hline & \multicolumn{3}{|c|}{ High school } & \multicolumn{3}{|c|}{ College } & \multicolumn{3}{|c|}{$>$ College } \\
\hline & All & Men & Women & All & Men & Women & All & Men & Women \\
\hline \multicolumn{10}{|l|}{ BNT } \\
\hline$N$ & 216 & 81 & 127 & 215 & 120 & 95 & 199 & 100 & 99 \\
\hline Mean & 32.51 & 32.25 & 32.69 & 33.96 & 33.83 & 34.14 & 34.29 & 34.6 & 33.97 \\
\hline$S D$ & 2.74 & 3.02 & 2.54 & 1.99 & 2.19 & 1.70 & 1.93 & 1.96 & 1.86 \\
\hline Upper Q & 34 & 34 & 34 & 35 & 36 & 35 & 36 & 36 & 35 \\
\hline Lower Q & 31 & 31 & 32 & 33 & 33 & 33 & 34 & 34 & 33 \\
\hline $5 \%$ & 28 & 28 & 28 & 30 & 29 & 31 & 30 & 29.5 & 30 \\
\hline \multicolumn{10}{|l|}{ Hooper } \\
\hline$N$ & 208 & 81 & 127 & 215 & 120 & 95 & 199 & 100 & 99 \\
\hline Mean & 25.16 & 24.36 & 25.67 & 25.54 & 25.27 & 25.89 & 26.03 & 25.93 & 26.13 \\
\hline$S D$ & 3.30 & 4.12 & 2.53 & 2.69 & 2.84 & 2.45 & 2.60 & 2.57 & 2.64 \\
\hline Upper Q & 27 & 26.5 & 27 & 27.5 & 27 & 27.5 & 28 & 28 & 28 \\
\hline Lower Q & 24 & 23.5 & 24.5 & 24.5 & 24 & 24.5 & 24.5 & 24.5 & 25 \\
\hline $5 \%$ & 18.5 & 17.5 & 20 & 20.5 & 19.5 & 21 & 21 & 21.25 & 21 \\
\hline \multicolumn{10}{|l|}{ FT-D } \\
\hline$N$ & 202 & 78 & 124 & 195 & 108 & 87 & 190 & 93 & 97 \\
\hline Mean & 44.98 & 48.27 & 42.91 & 47.32 & 50.07 & 43.90 & 47.17 & 50.46 & 44.01 \\
\hline$S D$ & 7.71 & 8.46 & 6.41 & 7.97 & 6.56 & 8.26 & 8.45 & 7.88 & 7.79 \\
\hline Upper Q & 50 & 53 & 46.5 & 53 & 54.5 & 50 & 53 & 56 & 49 \\
\hline Lower Q & 40 & 44 & 39 & 42 & 46.5 & 37 & 42 & 46 & 39 \\
\hline $5 \%$ & 32 & 32 & 32 & 34 & 38 & 31 & 32 & 36 & 30 \\
\hline \multicolumn{10}{|l|}{ WRAT } \\
\hline$N$ & 207 & 80 & 127 & 214 & 119 & 95 & 199 & 100 & 99 \\
\hline Mean & 45.80 & 43.88 & 47.01 & 49.60 & 49.13 & 50.18 & 51.28 & 51.41 & 51.14 \\
\hline$S D$ & 5.12 & 5.54 & 4.45 & 4.58 & 4.84 & 4.18 & 3.76 & 3.75 & 3.78 \\
\hline Upper Q & 49 & 47 & 50 & 54 & 54 & 54 & 55 & 54 & 55 \\
\hline Lower Q & 43 & 41 & 44 & 47 & 46 & 48 & 49 & 49 & 49 \\
\hline $5 \%$ & 37 & 34 & 39 & 42 & 41 & 43 & 44 & 44.5 & 44 \\
\hline \multicolumn{10}{|c|}{ 65- to 74-year-old age group } \\
\hline$N$ & 239 & 106 & 133 & 142 & 75 & 67 & 137 & 60 & 77 \\
\hline Mean & 10.00 & 9.81 & 10.16 & 11.25 & 11.05 & 11.46 & 11.97 & 11.93 & 12 \\
\hline$S D$ & 3.36 & 3.24 & 3.46 & 3.28 & 3.18 & 3.39 & 3.67 & 3.66 & 3.70 \\
\hline Upper Q & 12 & 12 & 12 & 14 & 13 & 14 & 15 & 14.5 & 15 \\
\hline Lower Q & 8 & 8 & 8 & 9 & 9 & 9 & 9 & 9 & 9 \\
\hline $5 \%$ & 4 & 4 & 4 & 6 & 6 & 4 & 6 & 5.5 & 6 \\
\hline \multicolumn{10}{|l|}{ LM-DR } \\
\hline$N$ & 239 & 106 & 133 & 142 & 75 & 67 & 137 & 60 & 77 \\
\hline Mean & 8.77 & 8.62 & 8.89 & 10.41 & 10.29 & 10.54 & 10.81 & 10.52 & 11.04 \\
\hline$S D$ & 3.60 & 3.35 & 3.8 & 3.54 & 3.29 & 3.82 & 3.68 & 3.47 & 3.84 \\
\hline Upper Q & 11 & 10 & 11 & 12 & 12 & 13 & 14 & 13 & 14 \\
\hline Lower Q & 7 & 7 & 7 & 8 & 8 & 8 & 8 & 8 & 8 \\
\hline $5 \%$ & 2 & 2 & 2 & 5 & 5 & 1 & 5 & 5 & 4 \\
\hline
\end{tabular}


TABLE 7 Continued

\begin{tabular}{|c|c|c|c|c|c|c|c|c|c|}
\hline & \multicolumn{3}{|c|}{ High school } & \multicolumn{3}{|c|}{ College } & \multicolumn{3}{|c|}{$>$ College } \\
\hline & All & Men & Women & All & Men & Women & All & Men & Women \\
\hline \multicolumn{10}{|l|}{ LM-\% } \\
\hline$N$ & 239 & 106 & 133 & 142 & 75 & 67 & 137 & 60 & 77 \\
\hline Mean & 86.44 & 87.54 & 85.57 & 91.43 & 93.49 & 89.11 & 90.43 & 89.13 & 91.45 \\
\hline$S D$ & 24.21 & 23.64 & 24.71 & 23.13 & 21.57 & 24.72 & 15.69 & 16.23 & 15.29 \\
\hline Upper Q & 100 & 100 & 100 & 100 & 100 & 100 & 100 & 100 & 100 \\
\hline Lower Q & 77.78 & 77.78 & 77.78 & 80 & 80 & 80 & 80 & 76.92 & 82.35 \\
\hline $5 \%$ & 50 & 50 & 50 & 66.67 & 69.23 & 25 & 62.5 & 61.25 & 66.67 \\
\hline \multicolumn{10}{|l|}{ VR-IR } \\
\hline$N$ & 240 & 107 & 133 & 142 & 75 & 67 & 137 & 60 & 77 \\
\hline Mean & 7.11 & 7.55 & 6.75 & 9.01 & 8.56 & 9.52 & 8.44 & 8.5 & 8.39 \\
\hline$S D$ & 3.09 & 3.25 & 2.92 & 2.79 & 2.62 & 2.9 & 3.17 & 3.25 & 3.13 \\
\hline Upper Q & 9 & 10 & 9 & 11 & 10 & 12 & 11 & 11 & 11 \\
\hline Lower Q & 5 & 5 & 5 & 7 & 7 & 7 & 6 & 6 & 6 \\
\hline $5 \%$ & 2 & 2 & 2 & 4 & 4 & 5 & 3 & 3 & 3 \\
\hline \multicolumn{10}{|l|}{ VR-DR } \\
\hline$N$ & 240 & 107 & 133 & 142 & 75 & 67 & 137 & 60 & 77 \\
\hline Mean & 6.11 & 6.40 & 5.87 & 7.81 & 7.41 & 8.25 & 7.52 & 7.88 & 7.23 \\
\hline$S D$ & 3.02 & 3.17 & 2.89 & 3.14 & 2.87 & 3.39 & 3.34 & 3.36 & 3.31 \\
\hline Upper Q & 8 & 9 & 8 & 10 & 9 & 11 & 10 & 10.5 & 10 \\
\hline Lower Q & 4 & 4 & 4 & 6 & 6 & 6 & 5 & 6 & 5 \\
\hline $5 \%$ & 1 & 1 & 1 & 2 & 2 & 2 & 3 & 2.5 & 3 \\
\hline \multicolumn{10}{|l|}{ VR-\% } \\
\hline$N$ & 240 & 107 & 133 & 142 & 75 & 67 & 136 & 59 & 77 \\
\hline Mean & 88.79 & 86.99 & 90.24 & 86.87 & 87.58 & 86.08 & 88.9 & 92.49 & 86.16 \\
\hline$S D$ & 40.30 & 30.39 & 46.84 & 25.94 & 24.30 & 24.83 & 24.44 & 19.46 & 27.47 \\
\hline Upper Q & 100 & 100 & 100 & 100 & 100 & 100 & 100 & 100 & 100 \\
\hline Lower Q & 70 & 66.67 & 71.43 & 75 & 75 & 75 & 75 & 75 & 66.67 \\
\hline $5 \%$ & 36.67 & 42.86 & 33.33 & 38.46 & 40 & 28.57 & 50 & 62.5 & 40 \\
\hline \multicolumn{10}{|l|}{ PA-TS } \\
\hline$N$ & 238 & 106 & 132 & 141 & 75 & 66 & 134 & 59 & 75 \\
\hline Mean & 12.82 & 12.08 & 13.42 & 13.21 & 12.29 & 14.24 & 13.88 & 13.24 & 14.39 \\
\hline$S D$ & 2.96 & 2.81 & 2.96 & 3.32 & 3.01 & 3.38 & 3.34 & 3.45 & 3.19 \\
\hline Upper Q & 15 & 14 & 16 & 16 & 15 & 17 & 16 & 15 & 17 \\
\hline Lower Q & 10 & 10 & 11 & 11 & 10 & 12 & 11 & 11 & 12 \\
\hline $5 \%$ & 8 & 8 & 9 & 8 & 8 & 9 & 9 & 8 & 9 \\
\hline \multicolumn{10}{|l|}{ Trails A } \\
\hline$N$ & 240 & 107 & 133 & 142 & 75 & 67 & 137 & 60 & 77 \\
\hline Mean & 38.24 & 38.14 & 38.32 & 35.27 & 36.81 & 33.54 & 37.85 & 39.05 & 36.91 \\
\hline$S D$ & 14.51 & 13.49 & 15.33 & 13.18 & 15.67 & 9.49 & 15.43 & 18.17 & 12.95 \\
\hline Upper Q & 43.5 & 44 & 43 & 40 & 43 & 39 & 45 & 48 & 42 \\
\hline Lower Q & 29 & 29 & 29 & 28 & 27 & 28 & 28 & 25.5 & 29 \\
\hline $5 \%$ & 22 & 21 & 22 & 20 & 21 & 19 & 20 & 21.5 & 20 \\
\hline
\end{tabular}


TABLE 7 Continued

\begin{tabular}{|c|c|c|c|c|c|c|c|c|c|}
\hline & \multicolumn{3}{|c|}{ High school } & \multicolumn{3}{|c|}{ College } & \multicolumn{3}{|c|}{$>$ College } \\
\hline & All & Men & Women & All & Men & Women & All & Men & Women \\
\hline \multicolumn{10}{|l|}{ Trails B } \\
\hline$N$ & 229 & 102 & 127 & 142 & 75 & 67 & 136 & 59 & 77 \\
\hline Mean & 102.52 & 102.12 & 102.84 & 90.89 & 89.41 & 92.54 & 86.46 & 88.15 & 85.17 \\
\hline$S D$ & 49.42 & 48.00 & 50.71 & 43.24 & 37.66 & 48.98 & 32.72 & 39.14 & 26.99 \\
\hline Upper Q & 118 & 118 & 115 & 103 & 102 & 105 & 98.5 & 106 & 95 \\
\hline Lower Q & 70 & 71 & 69 & 62 & 62 & 60 & 65 & 62 & 68 \\
\hline $5 \%$ & 52 & 52 & 52 & 48 & 49 & 48 & 49 & 45 & 54 \\
\hline \multicolumn{10}{|l|}{ Sim } \\
\hline$N$ & 239 & 107 & 132 & 142 & 75 & 67 & 137 & 60 & 77 \\
\hline Mean & 14.21 & 14.08 & 14.30 & 16.45 & 16.12 & 16.82 & 17.28 & 17.95 & 16.77 \\
\hline$S D$ & 3.57 & 4.06 & 3.14 & 3.29 & 3.45 & 3.09 & 3.45 & 3.44 & 3.38 \\
\hline Upper Q & 17 & 17 & 17 & 19 & 19 & 19 & 20 & 20.5 & 19 \\
\hline Lower Q & 12 & 11 & 13 & 14 & 14 & 15 & 15 & 16 & 15 \\
\hline $5 \%$ & 8 & 8 & 9 & 11 & 9 & 12 & 11 & 12 & 11 \\
\hline \multicolumn{10}{|l|}{ BNT } \\
\hline$N$ & 240 & 107 & 133 & 142 & 75 & 67 & 137 & 60 & 77 \\
\hline Mean & 31.65 & 32.45 & 31.02 & 33.42 & 33.55 & 33.28 & 32.97 & 33.55 & 32.52 \\
\hline$S D$ & 2.99 & 2.50 & 3.21 & 2.42 & 2.21 & 2.64 & 2.72 & 2.59 & 2.76 \\
\hline Upper Q & 34 & 34 & 33 & 35 & 35 & 35 & 35 & 35 & 35 \\
\hline Lower Q & 30 & 31 & 29 & 32 & 32 & 32 & 31 & 33 & 31 \\
\hline $5 \%$ & 26 & 27 & 25 & 30 & 30 & 30 & 28 & 28 & 28 \\
\hline \multicolumn{10}{|l|}{ Hooper } \\
\hline$N$ & 238 & 106 & 132 & 142 & 75 & 67 & 137 & 60 & 77 \\
\hline Mean & 23.75 & 23.52 & 23.94 & 25.51 & 24.07 & 25.01 & 24.31 & 23.43 & 25.01 \\
\hline$S D$ & 3.34 & 3.5 & 3.21 & 2.76 & 2.58 & 2.89 & 3.71 & 4.38 & 2.94 \\
\hline Upper Q & 26 & 26 & 26.5 & 26 & 26 & 27 & 27 & 26.5 & 27 \\
\hline Lower Q & 22 & 22 & 22 & 23.5 & 23 & 24.5 & 23 & 21.75 & 24 \\
\hline $5 \%$ & 17.5 & 17 & 17.5 & 18.5 & 18 & 19.5 & 17.5 & 16.25 & 19 \\
\hline \multicolumn{10}{|l|}{ FT-D } \\
\hline$N$ & 224 & 97 & 127 & 132 & 69 & 63 & 126 & 54 & 72 \\
\hline Mean & 42.17 & 46.02 & 39.24 & 44.48 & 47.09 & 41.62 & 43.10 & 48.15 & 39.3 \\
\hline$S D$ & 8.60 & 8.11 & 7.8 & 8.28 & 7.95 & 7.72 & 8.85 & 7.28 & 8.02 \\
\hline Upper Q & 48 & 52 & 45 & 50 & 52 & 48 & 49 & 53 & 44 \\
\hline Lower Q & 37 & 40 & 33 & 39 & 42 & 35 & 37 & 44 & 34 \\
\hline $5 \%$ & 28 & 33 & 28 & 29 & 30 & 29 & 29 & 35 & 26 \\
\hline \multicolumn{10}{|l|}{ WRAT } \\
\hline$N$ & 235 & 104 & 131 & 142 & 75 & 67 & 137 & 60 & 77 \\
\hline Mean & 44.72 & 43.55 & 45.66 & 49.61 & 48.55 & 50.81 & 50.35 & 50.45 & 50.27 \\
\hline$S D$ & 4.65 & 4.71 & 4.41 & 3.85 & 3.85 & 3.52 & 4.50 & 4.67 & 4.39 \\
\hline Upper Q & 48 & 47 & 48 & 53 & 51 & 53 & 54 & 54 & 54 \\
\hline Lower Q & 42 & 40 & 43 & 47 & 46 & 48 & 47 & 47.5 & 47 \\
\hline $5 \%$ & 36 & 35 & 38 & 44 & 42 & 45 & 42 & 41.5 & 43 \\
\hline
\end{tabular}


TABLE 7 Continued

\begin{tabular}{|c|c|c|c|c|c|c|c|c|c|}
\hline & \multicolumn{3}{|c|}{ High school } & \multicolumn{3}{|c|}{ College } & \multicolumn{3}{|c|}{$>$ College } \\
\hline & All & Men & Women & All & Men & Women & All & Men & Women \\
\hline \multicolumn{10}{|c|}{ 75+-year-old age group } \\
\hline \multicolumn{10}{|c|}{ LM-IR } \\
\hline$N$ & 117 & 53 & 64 & 57 & 27 & 30 & 43 & 17 & 26 \\
\hline Mean & 9.33 & 8.66 & 9.89 & 10.53 & 9 & 11.9 & 10.05 & 9.65 & 10.31 \\
\hline$S D$ & 3.75 & 4.01 & 3.45 & 3.24 & 2.45 & 3.28 & 3.81 & 4.43 & 3.41 \\
\hline Upper Q & 12 & 12 & 12 & 13 & 10 & 14 & 13 & 13 & 13 \\
\hline Lower Q & 7 & 6 & 7.5 & 8 & 8 & 10 & 7 & 5 & 8 \\
\hline $5 \%$ & 2 & 2 & 4 & 5 & 5 & 5 & 4 & 2 & 6 \\
\hline \multicolumn{10}{|l|}{ LM-DR } \\
\hline$N$ & 116 & 52 & 64 & 57 & 27 & 30 & 43 & 17 & 26 \\
\hline Mean & 8.19 & 7.48 & 8.77 & 9.35 & 8.11 & 10.47 & 8.67 & 8.00 & 9.12 \\
\hline$S D$ & 4.07 & 4.00 & 4.07 & 3.38 & 2.62 & 3.63 & 4.34 & 4.57 & 4.22 \\
\hline Upper Q & 11 & 11 & 12 & 12 & 10 & 13 & 12 & 12 & 13 \\
\hline Lower Q & 5.5 & 5 & 6.5 & 7 & 6 & 8 & 5 & 4 & 6 \\
\hline $5 \%$ & 0 & 0 & 1 & 4 & 4 & 4 & 1 & 0 & 3 \\
\hline \multicolumn{10}{|l|}{ LM-\% } \\
\hline$N$ & 114 & 51 & 63 & 57 & 27 & 30 & 43 & 17 & 26 \\
\hline Mean & 84.91 & 83.23 & 86.28 & 88.44 & 89.97 & 87.06 & 82.11 & 77.72 & 84.98 \\
\hline$S D$ & 29.96 & 33.95 & 26.50 & 16.97 & 17.23 & 16.9 & 29.74 & 37.55 & 23.71 \\
\hline Upper Q & 100 & 100 & 100 & 100 & 100 & 100 & 100 & 100 & 100 \\
\hline Lower Q & 75 & 66.67 & 76.92 & 80 & 80 & 76.92 & 66.67 & 66.67 & 66.67 \\
\hline $5 \%$ & 0 & 0 & 50 & 53.85 & 62.5 & 50 & 33.33 & 0 & 37.5 \\
\hline \multicolumn{10}{|l|}{ VR-IR } \\
\hline$N$ & 116 & 52 & 64 & 57 & 27 & 30 & 43 & 17 & 26 \\
\hline Mean & 5.97 & 6.02 & 5.92 & 7.25 & 7.93 & 6.63 & 7.05 & 7.76 & 6.58 \\
\hline$S D$ & 3.04 & 3.22 & 2.90 & 2.75 & 2.92 & 2.47 & 3.24 & 3.46 & 3.06 \\
\hline Upper Q & 8 & 8 & 8 & 9 & 10 & 9 & 9 & 9 & 7 \\
\hline Lower Q & 3 & 3 & 3 & 5 & 5 & 4 & 4 & 7 & 4 \\
\hline $5 \%$ & 2 & 1 & 2 & 3 & 4 & 3 & 3 & 1 & 3 \\
\hline \multicolumn{10}{|l|}{ VR-DR } \\
\hline$N$ & 115 & 51 & 64 & 57 & 27 & 30 & 43 & 17 & 26 \\
\hline Mean & 4.95 & 5.12 & 4.81 & 6.26 & 7.22 & 5.4 & 5.86 & 6.53 & 5.42 \\
\hline$S D$ & 3.24 & 3.39 & 3.13 & 2.91 & 2.85 & 2.72 & 3.62 & 3.86 & 3.45 \\
\hline Upper Q & 7 & 7 & 7 & 8 & 9 & 7 & 8 & 8 & 8 \\
\hline Lower Q & 2 & 3 & 2 & 4 & 5 & 4 & 3 & 4 & 3 \\
\hline $5 \%$ & 0 & 0 & 0 & 2 & 3 & 1 & 1 & 1 & 1 \\
\hline \multicolumn{10}{|l|}{ VR-\% } \\
\hline$N$ & 113 & 49 & 64 & 57 & 27 & 30 & 43 & 17 & 26 \\
\hline Mean & 79.24 & 82.07 & 77.08 & 87.53 & 93.10 & 82.51 & 80.69 & 81.56 & 80.12 \\
\hline$S D$ & 33.61 & 31.37 & 35.31 & 29.02 & 24.01 & 32.48 & 30.65 & 25.63 & 34.01 \\
\hline Upper Q & 100 & 100 & 100 & 100 & 112.5 & 100 & 100 & 100 & 100 \\
\hline Lower Q & 60 & 66.67 & 50 & 70 & 75 & 66.67 & 57.14 & 57.14 & 60 \\
\hline $5 \%$ & 0 & 18.18 & 0 & 33.33 & 53.85 & 33.33 & 33.33 & 42.86 & 25 \\
\hline
\end{tabular}


TABLE 7 Continued

\begin{tabular}{|c|c|c|c|c|c|c|c|c|c|}
\hline & \multicolumn{3}{|c|}{ High school } & \multicolumn{3}{|c|}{ College } & \multicolumn{3}{|c|}{$>$ College } \\
\hline & All & Men & Women & All & Men & Women & All & Men & Women \\
\hline \multicolumn{10}{|l|}{ PA-TS } \\
\hline$N$ & 116 & 52 & 64 & 56 & 27 & 29 & 43 & 17 & 26 \\
\hline Mean & 11.36 & 10.5 & 12.06 & 12.02 & 10.63 & 13.31 & 12.02 & 11.65 & 12.27 \\
\hline$S D$ & 3.24 & 3.12 & 3.18 & 3.04 & 2.27 & 3.13 & 3.09 & 3.04 & 3.16 \\
\hline Upper Q & 14 & 13 & 14.5 & 14 & 12 & 16 & 14 & 14 & 13 \\
\hline Lower Q & 9 & 8 & 10 & 10 & 9 & 11 & 10 & 10 & 10 \\
\hline $5 \%$ & 7 & 6 & 7 & 8 & 8 & 8 & 7 & 7 & 8 \\
\hline \multicolumn{10}{|l|}{ Trails A } \\
\hline$N$ & 112 & 49 & 63 & 56 & 27 & 29 & 43 & 17 & 26 \\
\hline Mean & 48.03 & 53.71 & 43.6 & 39.96 & 39.81 & 40.1 & 46.53 & 49.94 & 44.31 \\
\hline$S D$ & 22.02 & 25.52 & 17.84 & 13.48 & 13.38 & 13.81 & 31.06 & 45.28 & 17.2 \\
\hline Upper Q & 54.5 & 63 & 51 & 48 & 48 & 48 & 54 & 47 & 57 \\
\hline Lower Q & 34 & 34 & 33 & 29.5 & 30 & 29 & 31 & 30 & 33 \\
\hline $5 \%$ & 25 & 27 & 24 & 23 & 23 & 24 & 24 & 22 & 24 \\
\hline \multicolumn{10}{|l|}{ Trails B } \\
\hline$N$ & 106 & 48 & 58 & 54 & 27 & 27 & 41 & 17 & 24 \\
\hline Mean & 139.66 & 161.90 & 121.26 & 112.07 & 107.6 & 116.56 & 110.27 & 108 & 111.88 \\
\hline$S D$ & 73.12 & 89.83 & 49.35 & 53.08 & 43.25 & 61.9 & 56.46 & 61.9 & 53.59 \\
\hline Upper Q & 160.00 & 198 & 139 & 135 & 134 & 146 & 124 & 123 & 126 \\
\hline Lower Q & 92 & 92 & 89 & 79 & 79 & 77 & 74 & 66 & 84.5 \\
\hline $5 \%$ & 64 & 68 & 57 & 46 & 59 & 43 & 57 & 54 & 67 \\
\hline \multicolumn{10}{|l|}{ Sim } \\
\hline$N$ & 116 & 52 & 64 & 57 & 27 & 30 & 43 & 17 & 26 \\
\hline Mean & 13.30 & 12.87 & 13.66 & 16.33 & 16.30 & 16.37 & 16.21 & 17.29 & 15.50 \\
\hline$S D$ & 3.76 & 3.78 & 3.73 & 3.19 & 3.53 & 2.92 & 3.71 & 3.80 & 3.54 \\
\hline Upper Q & 16 & 16 & 16 & 18 & 20 & 18 & 20 & 20 & 18 \\
\hline Lower Q & 11 & 10.5 & 11.5 & 15 & 14 & 15 & 13 & 15 & 13 \\
\hline $5 \%$ & 6 & 6 & 7 & 9 & 11 & 9 & 11 & 8 & 11 \\
\hline \multicolumn{10}{|l|}{ BNT } \\
\hline$N$ & 115 & 51 & 64 & 57 & 27 & 30 & 42 & 17 & 25 \\
\hline Mean & 30.59 & 30.90 & 30.34 & 32.14 & 32.15 & 32.13 & 31.5 & 32.82 & 30.6 \\
\hline$S D$ & 4.11 & 4.57 & 3.71 & 3.08 & 2.88 & 3.31 & 3.72 & 3.26 & 3.80 \\
\hline Upper Q & 34 & 34 & 33 & 34 & 34 & 35 & 35 & 35 & 33 \\
\hline Lower Q & 28 & 29 & 28 & 31 & 31 & 31 & 29 & 31 & 28 \\
\hline $5 \%$ & 23 & 25 & 23 & 25 & 25 & 25 & 25 & 26 & 24 \\
\hline \multicolumn{10}{|l|}{ Hooper } \\
\hline$N$ & 112 & 50 & 62 & 57 & 27 & 30 & 42 & 17 & 25 \\
\hline Mean & 21.02 & 20.25 & 21.64 & 22.39 & 22.43 & 22.37 & 22.71 & 23.32 & 22.3 \\
\hline$S D$ & 4.35 & 4.85 & 3.84 & 3.40 & 3.69 & 3.17 & 3.31 & 3.15 & 3.41 \\
\hline Upper Q & 24.5 & 24.5 & 25 & 25 & 25.5 & 25 & 25 & 25 & 25 \\
\hline Lower Q & 18.25 & 18 & 19 & 20 & 20. & 20 & 20.5 & 22 & 20 \\
\hline $5 \%$ & 13.5 & 11 & 15 & 17.5 & 18.5 & 17.5 & 15.5 & 15 & 15.5 \\
\hline
\end{tabular}


TABLE 7 Continued

\begin{tabular}{|c|c|c|c|c|c|c|c|c|c|}
\hline & \multicolumn{3}{|c|}{ High school } & \multicolumn{3}{|c|}{ College } & \multicolumn{3}{|c|}{$>$ College } \\
\hline & All & Men & Women & All & Men & Women & All & Men & Women \\
\hline \multicolumn{10}{|l|}{ FT-D } \\
\hline$N$ & 105 & 47 & 58 & 49 & 25 & 24 & 36 & 15 & 21 \\
\hline Mean & 38.27 & 40.4 & 36.53 & 41.27 & 43.4 & 39.04 & 40.53 & 43.8 & 38.19 \\
\hline$S D$ & 9.78 & 9.95 & 9.36 & 8.52 & 9.08 & 7.43 & 6.95 & 5.1 & 7.26 \\
\hline Upper Q & 45 & 47 & 44 & 47 & 50 & 45 & 46 & 47 & 43 \\
\hline Lower Q & 32 & 34 & 29 & 34 & 39 & 33 & 36.5 & 40 & 33 \\
\hline $5 \%$ & 22 & 24 & 18 & 26 & 25 & 26 & 26 & 36 & 26 \\
\hline \multicolumn{10}{|l|}{ WRAT } \\
\hline$N$ & 112 & 51 & 61 & 57 & 27 & 30 & 42 & 16 & 26 \\
\hline Mean & 45.57 & 44.78 & 46.23 & 49.65 & 48.04 & 51.10 & 48.69 & 50.19 & 47.77 \\
\hline$S D$ & 4.78 & 4.58 & 4.87 & 4.29 & 4.52 & 3.57 & 4.72 & 5.64 & 3.89 \\
\hline Upper Q & 49 & 48 & 50 & 53 & 51 & 54 & 53 & 55 & 50 \\
\hline Lower Q & 42 & 42 & 43 & 47 & 46 & 49 & 45 & 45 & 45 \\
\hline $5 \%$ & 38 & 37 & 39 & 39 & 39 & 43 & 42 & 42 & 41 \\
\hline
\end{tabular}

The association between cognitive performance in the Offspring, with respect to age, education, and gender appeared remarkably similar to that reported in the Original Cohort by Elias et al., (1997). Increased age was associated with lower performance; higher education with better performance.

The Elias et al., (1997) study of the Original Cohort reported one significant three-way interaction; that is, the oldest men in the lowest education group scored significantly lower compared to all other groups. We did not find any significant three-way interaction effects in the Offspring. Our inability to replicate this finding, however, does not necessarily reflect a conflict in findings with our earlier study. Rather, we were unable to conduct a comparable analysis in this age/education group with the Offspring Cohort because we only had two subjects who fit the lowest educational category used in our Original Cohort study. The uniqueness of the Framingham Study is its use of a community-based sample, and in this case, our Offspring Cohort, when compared to our Original Cohort, represents the secular changes in educational attainment. Level of educational attainment is known to be positively correlated with performance on cognitive tests, and our results on the amount of variance explained by education provide additional confirmation of this phenomenon (see Table 5). Thus it follows that as the education level of a population rises, average scores on cognitive measures also increase.

Increased years of educational attainment, however, does not fully account for higher test performance. Our multivariable analysis 
comparing the Offspring and Original Cohorts revealed that Offspring test performance was consistently higher than that of the Original Cohort. Although as expected, age and education were statistically significant, they did not fully explain the difference in performance between cohorts. One possible explanation is that the education obtained by the Offspring is qualitatively different from that of their parents. It may be that qualitative changes in academic instruction as well as enhanced educational opportunities provided by television, radio, the internet, and other sources are not captured by years of education.

Higher economic status in the Offspring Cohort coupled with significant advances in public health and medical care may be two of a number of factors that are altering the impact of the aging process on cognitive functioning. Both of these societal developments lead to better nutrition, healthier lifestyles, use of preventive medical services, and advanced treatments for illnesses, which, in turn, attenuate the aging process depicted by the Original Cohort's generation. Although we have no direct evidence that this was the case in the present study, it is reasonable to speculate that these factors, in addition to education, influenced our findings. Although the Framingham Original Cohort and the Offspring Cohort studies provide a rich source of information on cardiovascular risk factors, it is difficult to compare the cohorts with respect to health status because a higher proportion of individuals displaying cardiovascular risk may well represent better detection methods rather than an increase in disease prevalence. Thus, we considered an evaluation of the role of health factors in the differences between the Original and the Offspring Cohorts beyond the scope of this study.

Although a fully definitive explanation is not possible, our finding that the Offspring Cohort performed significantly better on cognitive tests than their parents supports our contention that new norms are needed based on data from contemporary samples. The inability to control for factors associated with generational changes is further highlighted by the limitations associated with cross-sectional methodology. It is well-accepted that differences in age cohorts may be exaggerated or an incidental finding linked to factors unique to each age group.

Although this Offspring Study updates results from the Original Cohort, it too must be considered in light of its own limitations. Education continues to be a significant factor associated with cognitive performance, and we recognize that the lower education range is underrepresented in our population. Also, our subjects may be somewhat more health conscious than average because of their family's long-term involvement with the Framingham Study. Finally, we do not provide data that reflects the multiethnicity of the general population.

Despite these limitations, our study provides important insight into age-cohort differences in neuropsychological test performance. The 
immediate impact of our Offspring Study is to provide new information suggesting that normative data obtained from previous generations may not be representative of the postdepression generation. The long-term implication of our research is that if these trends continue, data reported in this study may be less applicable to generations subsequent to our Offspring Cohort. It is clear that we must continue surveillance of each generation, documenting the impact that unique educational, social, and economic variables have on their cognitive function. It is our hope that our normative data, based on a large community-based sample, will encourage other investigators to publish contemporary normative data that will be useful in constructing indices of cognitive deficit, including the various definitions of MCI now reported in the literature.

\section{REFERENCES}

Arceneaux, J. M., Kirkendall, D. J., Hill, S. K., Dean, R. S., \& Anderson, J. L. (1997). Validity and reliability of rapidly alternating movement tests. The International Journal of Neuroscience, 89, 281-286.

Bachman, D. L., Wolf, P. A., Linn, R, Knoefel, J. E., Cobb, J., Belanger, A. et al., (1992). Prevalence of dementia and probable senile dementia of the Alzheimer type in the Framingham Study. Neurology, 42, 115-119.

Birren, J. E. \& Morrison, D. F. (1961). Analysis of the WAIS subtests in relation to age and education. Journal of Gerontology, 16, 363-369.

Boyd, J. L. (1981). A validity study of the Hooper Visual Organization Test. Journal of Consulting and Clinical Psychology, 49, 15-19.

Bushell, C. M. \& Martin, A. (1997). Automatic semantic priming of nouns and verbs in patients with Alzheimer's disease. Neuropsychologia, 35, 1059-1067.

Caffarra, P., Scaglioni, A., Malvezzi, L., Girotti, F., Soliveri, P., Musicco, M. et al., (1991). Fuld's formula and WAIS subtests in differential diagnosis of dementia. Functional Neurology, 6, 23-27.

Cagney, K. A. \& Lauderdale, D. S. (2002). Education, wealth, and cognitive function in later life. Journal of Gerontology Series B. Psychological Science and Social Sciences, 57, 163-172.

Christensen, H., Korten, A. E., Jorm, A. F., Henderson, A. S., Jacomb, P. A., Rodgers, B., \& Mackinnon, A. J. (1997). Education and decline in cognitive performance: Compensatory but not protective. Internal Journal of Geriatric Psychiatry, 12, 323-330.

Doraiswamy, P. M., Steffens, D. C., Pitchumoni, S., \& Tabrizi, S. (1998). Early recognition of Alzheimer's disease: What is consensual? What is controversial? What is practical? Journal of Clinical Psychiatry, 59 (Suppl 13), 6-18.

Elias, M. F., Elias, P. K., D'Agostino, R. B., Silbershatz, H., \& Wolf, P. A. (1997). Role of age, education, and gender on cognitive performance in the Framingham Heart Study: Community-based norms. Experimental Aging Research, 23, 201-235.

Farmer, M. E., Kittner, S. J., Rae, D. S., Bartko, J. J., \& Regier, D. A. (1995). Education and change in cognitive function. The Epidemiologic Catchment Area Study. Annals of Epidemiology, 5, 1-7.

Filley, C. M. \& Cullum, C. M. (1997). Education and cognitive function in Alzheimer's disease. Neuropsychiatry, Neuropsychology, and Behavioral Neurology, 10, 48-51. 
Fleishman, D. A., Gabrieli, J. D., Rinaldi, J. A., Reminger, S. L., Grinnell, W. R., Lange, L. et al., (1997). Word-stem completion priming for perceptually and conceptually encoded words in patients with Alzheimer's disease. Neuropsychologia, 35, 25-35.

Frank, R., Wiederholt, W. C., Kritz-Silverstein, D. K., Salmon, D. P., \& Barrett-Connor, E. (1996). Effects of sequential neuropsychological testing of an elderly community-based sample. Neuroepidemiology, 15, 257-268.

Garrison, R. J., Kannel, W. B., Stokes, J., 3rd, \& Castelli, W. P. (1987). Incidence and precursors of hypertension in young adults: The Framingham Offspring Study. Preventive Medicine, 16, 235-251.

Gaudino, E. A., Geisler, M. W., \& Squires, N. K. (1995). Construct validity in the trial making test: what makes part b harder? Journal of Clinical \& Experimental Neuropsychology, $17(4), 525-535$.

Gauthier, S. (1999). Managing expectations in the long-term treatment of Alzheimer's disease. Gerontology, 45 (Suppl 1), 33-38.

Gerson, A. (1974). Validity and reliability of the Hooper Visual Organization Test. Perceptual and Motor Skills, 39, 95-100.

Gfeller, J. D., Meldrum, D. L., \& Jacobi, K. A. (1995). The impact of constructional impairment on the WMS-R Visual Reproduction subtests. Journal of Clinical Psychology, 51, $58-63$.

Giovagnoli, A. R., Del Pesce, M., Mascheroni, S., Simoncelli, M., Laiacona, M., \& Capitani, E. (1996). Trail making test: Normative values from 287 normal adult controls. Italian Journal of Neurological Sciences, 17, 305-309.

Johnstone, B. \& Wilhelm, K. L. (1996). The longitudinal stability of the WRAT-R Reading subtest: Is it an appropriate estimate of premorbid intelligence? Journal of the International Neuropsychological Society, 2, 282-285.

Lezak, M. D. (1983). Neuropsychological assessment, (2nd ed.). New York: Oxford University Press.

Mack, W. J., Freed, D. M., Williams, B. W., \& Henderson, V. W. (1992). Boston Naming Test: shortened versions for use in Alzheimer's disease. Journal of Gerontology, 47, $154-158$.

Manly, J. J., Jacobs, D. M., Sano, M., Bell, K., Merchant, C. A., Small, S. A., \& Stern, Y. (1999). Effect of literacy on neuropsychological test performance in nondemented, education-matched elders. Journal of International Neuropsychological Society, 5, 191-202.

Monti, L. A., Gabrieli, J. D., Wilson, R. S., Beckett, L. A., Grinnell, E., Lange, K. L. et al., (1997). Sources of priming in text rereading: intact implicit memory for new associations in older adults and in patients with Alzheimer's disease. Psychology and Aging, 12, $536-547$.

Petersen, R. C., Smith, G. E., Waring, S. C., Ivnik, R. I., Tangalos, E. G., \& Kokmen, E. (1999). Mild cognitive impairment: Clinical characterization and outcomes. Archives of Neurology, 56, 303-308.

Petersen, R. C., Doody, R., Kurz, A., Mohs, R., Morris, J. C., Rabins, P. V., Ritchie, K., Russor, M., Thal, L., \& Winblad, B. (2001). Current concepts in mild cognitive impairment. Archives of Neurology, 58, 1985-1992.

Reitan, R. M. \& Wolfson, D. (1995). Influence of age and education on neuropsychological test results. The Clinical Neuropsychologist, 9, 151-158.

Ruff, R. M. \& Parker. S. B. (1993). Gender- and age-specific changes in motor speed and eye-hand coordination in adults: Normative values for the Finger Tapping and Grooved Pegboard Tests. Perceptual and Motor Skills, 76 (3 Pt 2), 1219-1230.

Sacco, R. L., Wolf, P. A., Kannel, W. B., \& McNamara, P. M. (1982). Survival and recurrence following stroke. The Framingham study. Stroke, 13, 290-295. 
Schmand, B., Geerlings, M. I., Jonker, C. \& Lindeboom, J. (1998). Reading ability as an estimator of premorbid intelligence: Does it remain stable in emergent dementia? Journal of Clinicial Experimental Neuropsychology, 20(1), 42-51.

Shimoyama, I., Ninchoji, T., \& Uemura, K. (1990). The finger-tapping test. A quantitative analysis. Archives of Neurology, 47, 681-684.

Wiederholt, W. C., Cahn, D., Butters, N. M., Salmon, D. P., Kritz-Silverstein, D., \& Barrett-Connor, E. (1993). Effects of age, gender and education on selected neuropsychological tests in an elderly community cohort. Journal of the American Geriatric Society, 41, 639-647. 